\title{
Levels of anxiety, dominant tendency and mirror-tracing performance
}

MURRAY MEISELS, ZAKHOUR I. YOUSSEF AND MICHAEL J. DORAN EASTERN MICHIGAN UNIVERSITY

Farber \& Spence's (1953) demonstration that the drive theory of anxiety is useful in predicting motor performance was replicated, using a maze tracing task under simple and complex conditions. Results under the complex condition supported drive theory, while results under the simple condition were equivocal.

This experiment used a maze-tracing task to replicate the Farber \& Spence (1953) finding that the drive theory of anxiety is useful in predicting motor performance. Under the simple condition the $\mathrm{S}$ traced a long alley maze, as in everyday writing, and the dominant tracing tendency was, by definition, correct. For the complex condition the maze was placed in a mirror apparatus. Since this condition required the reversal of normal writing habits, then the dominant tendency, which was to draw as in normal conditions, was incorrect. The primary predictions, derived from drive theory, were that high anxious Ss would perform better on the simple task but would perform worse on the initial trials of the complex task.

Method

The Taylor Manifest Anxiety Scale (Taylor, 1953) was administered to 161 introductory psychology students at Eastern Michigan University. From the extreme upper and lower $18 \%$ of the continuum of scores 15 high anxious (HA) and 15 low anxious (LA) Ss were selected. Because some Ss failed to appear, however, only $12 \mathrm{HA}$ and $14 \mathrm{LA}$ Ss were run, and the analyses were completed on these Ss. S's task was to trace the outline of a $1 / 4 \mathrm{in}$. wide maze which consisted of 29 angular turns and four arcs. A mirrortracing board, with appropriate mirror and shield, and a stopwatch for timekeeping purposes, remained on the table of the experimental cubicle at all times. The E, who did not know whether $S$ was from the HA or LA group, introduced the task as an inquiry into mirror-tracing performance. $S$ was told that speed and accuracy were the properties of a good performance. $S$ received six trials under the simple condition, followed by 15 trials under the complex or mirror condition, and then, an additional two trials on the simple task. The two indices of performance were speed and number of errors. Errors were scored when S's pencil either touched or passed through the edge of the maze; an additional error was scored for each $1 / 2$ in. that S's pencil remained outside the maze, and for each time $S$ lifted his pencil from the test protocol.

\section{Results}

When tracing the maze under nonmirror conditions, mean differences between HA and LA groups on any given trial for either the time or error variable were very slight. For the error variable, the HA mean was lower than the LA mean in seven of eight trials $\left(X^{2}=9.00, d f=1, p<.01\right)$, indicating better performance for the HA group as predicted. For the time variable, however, the LA mean was lower than the HA mean on all eight trials $\left(X^{2}=16.00, p<.001\right)$, indicating better performance for the LA group which was directly opposed to prediction.

The prediction that H.A Ss would initially perform worse on the complex task was supported by twotailed $t$ test comparisons between HA and LA groups on all 15 mirror-tracing trials. For errors, significant differences obtained only on Trials 3 and $4(t=2.60$ and 2.23, respectively, $d f=24, p<.02$ and $<.05$, respectively), with the LA group indicating significantly better performance in each instance. For the speed variable significant differences obtained on Trials 3 and $5(t=2.56$ and 2.68, respectively, $p<.02)$, with Trial 4 significant at the .10 level $(t=1.73)$, and the scores indicating better performance for the LA group. The major hypothesis of the study was also tested by analysis of variance comparing performance on the first four trials on the simple vs complex tasks as a within factor and anxiety level as the between factor. For errors, as predicted, dominance of task interacted with anxiety level $(F=5.54, \mathrm{df}=1 / 24, \mathrm{p}<$ $.05)$, with HA Ss performing better on the simple task and poorer on the complex task. The results of the analysis for time, though in the predicted direction, failed to achieve statistical significance $(F=2.35)$.

A further prediction from drive theory is that on a complex task the performance of HA Ss will eventually equal or exceed that of LA Ss as response dominance changes in the hierarchy. This prediction was tested by analysis of variance comparing performance on the first three trials vs middle three trials vs last three trials as a within factor; and high vs low anxiety as the between factor (see Table 1). For errors, as predicted, anxiety interacted with trials $(\mathrm{F}=4.18, \mathrm{df}=2 / 48, \mathrm{p}<.05)$, with the HA group mani-

Table 1 Mean scores on three trial blocks of mirror tracing for HA and LA groups

\begin{tabular}{|c|c|c|c|c|c|c|c|}
\hline & \multicolumn{3}{|c|}{ ERRORS ON TRIALS } & \multicolumn{3}{|c|}{ TIME ON TRIALS (sec) } \\
\hline & & $1-3$ & $7-9$ & $13-15$ & $1-3$ & $7-9$ & $13-15$ \\
\hline LA & $\begin{array}{l}M \\
S D\end{array}$ & $\begin{array}{l}91.9 \\
31.5\end{array}$ & $\begin{array}{l}48.2 \\
23.2\end{array}$ & $\begin{array}{l}36.4 \\
16.0\end{array}$ & $\begin{array}{l}419.0 \\
195.0\end{array}$ & $\begin{array}{r}175.5 \\
61.6\end{array}$ & $\begin{array}{r}142.0 \\
41.4\end{array}$ \\
\hline HA & $\begin{array}{l}M \\
S D\end{array}$ & $\begin{array}{r}117.8 \\
38.1\end{array}$ & $\begin{array}{l}46.0 \\
22.3\end{array}$ & $\begin{array}{l}36.2 \\
17.3\end{array}$ & $\begin{array}{l}549.4 \\
257.5\end{array}$ & $\begin{array}{r}216.5 \\
77.5\end{array}$ & $\begin{array}{r}157.9 \\
50.4\end{array}$ \\
\hline
\end{tabular}


festing initially poorer performance but then equaling (but not exceeding) the performance of the LA group. For the time variable, however, the interaction effect failed to achieve statistical significance, and the prediction was thus confirmed only for errors.

Discussion

The equivocal results for the simple condition, with HA Ss making significantly fewer errors but taking significantly more time, precludes any conclusion regarding which group performed better on this task. The correlation coefficient between errors and time (on Trial 1 of the simple condition for all $26 \mathrm{Ss}$ ) was $-.62(p<.001)$, indicating that $\mathrm{Ss}$ either focused on making few errors at the expense of time, or vice versa. This makes the results uninterpretable for drive theory since good performance on one index meant poor performance on the other. These results are, however, consistent with previous findings that HA Ss tend to approach tasks in a more obsessivecompulsive or perfectionistic manner than do LA Ss (Sarason, 1960).

On the complex task the correlation between the two dependent variables for 26 Ss on Trial 1 was .45 $(p<.02)$, so that poor performance on one index meant poor performance on the other. The results here were consistent with drive theory, except that, due to large variabllity, significant interaction effects were not obtained for the speed factor. The possibility of an asymptote artifact influencing the interaction between anxiety and trials was negated by more recent data which indicated continued improvement in mirror tracing through at least 30 trials. The results under the complex condition, in sum, are consistent with the Farber \& Spence (1953) finding that drive theory is useful in predicting motor performance.

An additional finding of theoretical interest stemmed from comparison of the correlations between time and errors on the two tasks. On the simple task there was little response competition-it was sufficiently easy that accuracy was an inverse function of speed. On the early trials of the complex task when response competition was greatest the two dependent variables were positively correlated, but this correlation was vitiated by the middle trials, and became negative on later trials. These latter correlations reflect, of course, the extinguishing of the competing incorrect response. This suggests that response competition is a concomitant variable which alters the degree and/ or direction of correlation between two dependent variables.

\section{References}

FARBER, I. E., \& SPENCE, K. W. Complex learning and conditioning as a function of anxiety. J. exp. Psychol., 1953, 45, 120-125.

SARASON, I. G. Empirical findings and theoretical problems in the use of anxiety scales. Psychol. Bull., 1960, 57, 403-415.

TAYLOR, J. A. A personality scale of manifest anxiety.J. abnom. soc. Psychol., 1953, 48, 285-290. 